\title{
Effects of six functional SNPs on the urinary 8-isoprostane level in a general Japanese population; Shimane COHRE Study
}

\author{
Yoji Suyama $^{\mathrm{a}}$, Chikashi Matsuda ${ }^{\mathrm{a}}$, Minoru Isomura ${ }^{\mathrm{g}}$, Tsuyoshi Hamano ${ }^{\mathrm{f}}$, Kenji Karino $^{\mathrm{b}}$, \\ Masayuki Yamasaki ${ }^{\mathrm{c}}$, Shuhei Yamaguchi ${ }^{\mathrm{d}, \mathrm{f}}$, Kuninori Shiwaku ${ }^{\mathrm{d}, \mathrm{f}}$, Junichi Masuda ${ }^{\mathrm{a}, \mathrm{f}}$ and \\ Toru Nabika ${ }^{e, f, *}$ \\ ${ }^{a}$ Department of Laboratory Medicine, Shimane University School of Medicine, Izumo, Japan \\ ${ }^{\mathrm{b}}$ Department of Community Medicine Management, Shimane University School of Medicine, Izumo, Japan \\ ${ }^{\mathrm{c}}$ Department of Environmental and Preventive Medicine, Shimane University School of Medicine, Izumo, Japan \\ ${ }^{\mathrm{d}}$ The Third Department of Internal Medicine, Shimane University School of Medicine, Izumo, Japan \\ ${ }^{\mathrm{e}}$ Department of Functional Pathology, Shimane University School of Medicine, Izumo, Japan \\ ${ }^{\mathrm{f}}$ The Center of Health Research and Education (COHRE), Shimane University, Izumo, Japan \\ ${ }^{g}$ Department of Cancer Genomics, The Cancer Institute of the Japanese Foundation for Cancer Research, Tokyo, \\ Japan
}

\begin{abstract}
Oxidative stress is an important risk factor for cardiovascular diseases. Although a variety of genetic factors are assumed to contribute to the regulation of oxidative stress, evidence in human populations is insufficient. In this study, we therefore evaluated the effects of six functional single-nucleotide polymorphisms (SNPs) on the oxidative stress under a crosssectional study design. Participants of the health examination in two neighboring counties were recruited in a mountainous region of Shimane prefeture, Japan $(n=1092)$. As a marker for the oxidative stress, the urinary 8-isoprostane (IsoP) was measured by ELISA. The six SNPs were genotyped using the Taqman method. None of the SNPs showed a significant effect on the IsoP level. However, the Generalized Multiple Dimensionality Reduction (GMDR) method identified that the combination of the two SNPs, MTHFR C677T and eNOS T-786C, showed a significant effect on the IsoP level in this population. The linear regression analysis confirmed that the high risk genotype identified in the GMDR was an independent factor influencing the IsoP even after adjustment of confounding factors. This result suggested that GMDR analysis might be useful to identify concealed effects of combined SNPs.
\end{abstract}

Keywords: Oxidative stress, generalized multiple dimensionality reduction, SNPs, 8-isoprostane

\section{Introduction}

Oxidative stress is one of important risk factors for various cardiovascular diseases [1]. Many environmental and genetic factors are suggested to influence the oxidative stress, which, in turn, are inferred in the patho-

* Corresponding author: Toru Nabika, Department of Functional Pathology, School of Medicine Shimane University, Izumo 6938501, Japan. Tel.: +81 85320 2136; Fax: +81 85320 2135; E-mail: nabika@med.shimane-u.ac.jp. genesis of atherosclerosis, coronary heart diseases and cerebrovascular diseases [2-4].

Functional SNPs have been identified in the genes encoding enzymes regulating oxidative stress response [5-10]. Although the biological significance of those SNPs was confirmed in functional assays evaluating the promoter and the enzyme activity as well as quantity of the mRNA and of the enzyme, relative lack of information on the effects of such SNPs on the level of oxidative stress in the human population is apparent.

In this study, we therefore performed a crosssectional analysis on effects of SNPs on the urinary 8- 
isoprostane (IsoP) level, a marker for oxidative stress, to examine a hypothesis that some functional SNPs affect oxidative stress in the general population. IsoP is an isomer of prostaglandin $\mathrm{F} 2 \alpha$, which is generated through non-enzymatic peroxidation of arachidonic acid by the oxidative stress [11]. This substance is stable in the urine and is a good marker for the oxidative stress under the epidemiological study design [12-15].

Six functional SNPs were evaluated in the study; they are T-107C and Q192R in the paraoxonase 1 (PON1), T-786C in the endothelial nitric oxide synthase (eNOS), C677T in the methylenetetrahydrofolate reductase (MTHFR), G994T in the lipoproteinassociated phospholipase A2 (LpPLA2) and C242T in the $\mathrm{p} 22$ phox gene, all of which were shown to regulate either the promoter activity (eNOS T-786C and PON1 T-107C), the enzyme activity (PON1 Q192R, LpPLA2 G994T and p22phox C242T), or the stability of the enzyme (MTHFR C677T) in the previous studies [5-10]. In addition to analyzing individual SNPs as independent risk factors, we evaluated gene-gene interaction among the SNPs studied. To accomplish this, Generalized Multifactor Dimensionality Reduction (GMDR) analysis was employed, which is a statistical method newly introduced to deal with complex gene-gene interaction [16].

We report here that, while no individual SNPs had significant effects, the combination of the 2 SNPs showed a significant effect on the IsoP level.

\section{Methods}

\subsection{Subjects}

Participants were recruited from the health examinations performed in two neighboring counties ( $\mathrm{K}$ and $\mathrm{M}$ counties) in a rural area of Shimane Prefecture, Japan in 2006 and 2007. Smoking and drinking behavior, as well as the history of treatment of hypertension (HT), hyperlipidemia and diabetes mellitus (DM) were obtained in the interview. Blood pressure was measured with automatic sphygnomanometers after at least 15min rest in a sitting position. All the participants agreed to give a written informed consent. This study conformed with the Code of Ethics of the World Medical Association (Declaration of Helsinki), and was approved by the ethical committee of Shimane University School of Medicine.

\subsection{Laboratory examinations}

Blood and urine samples were collected after overnight fasting. Biochemical markers were measured in plasma or serum using an automated biochemical analyzer. Estimated glomerular filtration rate (eGFR) was calculated using a formula recommended by the Japanese Society of Nephrology [17]. Urine samples were kept frozen at $-80^{\circ} \mathrm{C}$ until the measurement of IsoP. The measurement of IsoP was done using an ELISA kit commercially available (Oxford Biomedical Research, Rochester Hills, MI, USA). Although gas chromatography mass spectrometry is the golden standard to measure IsoP, it is quite difficult to apply this method to the measurement of a thousand samples because it is too labor-intensive and time-consuming. The urinary IsoP level was standardized with urinary creatinine.

\subsection{SNP typing}

SNP typing was done by the Taqman method following the protocol provided by the supplier (Applied Biosystems, Foster City, CA, USA). Primers used in the analysis were listed in the supplement table. All the samples were genotyped twice to check the reproducibility. Subjects either with a low signal intensity or with discrepant genotypes between the two results were excluded from the analysis. Excluded subjects were less than $3 \%$ for each SNP.

\subsection{Statistics}

Mean and SEM or $95 \%$ confidence interval are shown for the measures. As the IsoP level, triglyceride (TG), high-sensitive C-reactive protein (CRP) and homeostasis model assessment-insulin resistance (HOMA-R) were highly skewed from the normal distribution, log-transformed values were used in the analysis. The Student's t-test, the contingency table analysis, the simple regression analysis with Pearson's $r$ and ANOVA were used in the univariate analyses when they were appropriate. The linear regression analysis was performed on the potential factors influencing the IsoP levels. All analyses were performed using JMP v.8.0 (SAS Institute Inc, Cary, NC, USA).

\subsection{GMDR analysis}

Effects of interaction among the 6 SNPs were tested using GMDR, which is an extended version of MDR $[18,19]$. The basic concept of MDR is to reduce 
Table 1

Demographic data of the two populations studied

\begin{tabular}{lccc}
\hline & K county & M county & $\mathrm{p}^{*}$ \\
\hline sex $(\mathrm{F} / \mathrm{M})$ & $422 / 257$ & $273 / 140$ & 0.19 \\
age & $71.0[70.3,71.7]$ & $67.2[66.4,68.1]$ & $<0.0001$ \\
$\mathrm{BMI}, \mathrm{Kg} / \mathrm{m}^{2}$ & $22.6[22.4,22.8]$ & $22.6[22.3,22.9]$ & 0.95 \\
$\mathrm{SBP}, \mathrm{mmHg}$ & $132[130,133]$ & $132[131,134]$ & 0.45 \\
$\mathrm{DBP}, \mathrm{mmHg}$ & $77[76,78]$ & $78[77,79]$ & 0.42 \\
$\mathrm{~T}-\mathrm{C}, \mathrm{mg} / \mathrm{dL}$ & $210[207,212]$ & $200[197,203]$ & $<0.0001$ \\
$\mathrm{HDL}-\mathrm{C}, \mathrm{mg} / \mathrm{dL}$ & $57.7[56.6,58.7]$ & $67.8[66.5,69.2]$ & $<0.0001$ \\
$\mathrm{LDL}-\mathrm{C}, \mathrm{mg} / \mathrm{dL}$ & $121[119,123]$ & $119[117,122]$ & 0.45 \\
TG, mg/dL & $93.3[89.1,95.9]$ & $97.7[95.5,102]$ & 0.023 \\
eGFR, mL/min/1.73m 2 & $73.2[72.1,74.4]$ & $80.4[78.9,81.9]$ & $<0.0001$ \\
HOMA-R & $0.64[0.60,0.68]$ & $0.75[0.70,0.81]$ & 0.001 \\
CRP, mg/dL & $0.056[0.051,0.060]$ & $0.054[0.049,0.060]$ & 0.7 \\
IsoP, ng/mg Cre & $1.33[1.27,1.39]$ & $1.56[1.48,1.65]$ & $<0.0001$ \\
Current smoker, \% & 6.9 & 5.3 & 0.28 \\
Regular Drinker, \% & 19.4 & 25.9 & 0.006 \\
Medication for & & & \\
hypertension, \% & 40.7 & 32.3 & 0.02 \\
hyperlipidemia, \% & 15.3 & 13.1 & 0.61 \\
diabetes, \% & 5.5 & 4.4 & 0.73 \\
\hline
\end{tabular}

Means and $95 \%$ confidence intervals of the mean are indicated for continuous variables. BMI; body-mass index, SBP; systolic blood pressure, DBP; diastolic blood pressure, T$\mathrm{C}$; total cholesterol, HDL-C; high-density lipoprotein cholesterol, LDL-C; low-density lipoprotein cholesterol, TG; triglyceride, eGFR; estimated glomerular filtration rate, HOMA-R; homeostasis model assessment-insulin resistant, CRP; C-reactive protein, IsoP; 8-isoprostane, *: by Student's t-test or $\chi 2$ test.

a multi-dimensional set of multilocus genotypes [for example, 6 SNPs (having 3 genotypes) generate $3^{6}$ (= 729) combinations of genotypes] to one dimension by categorizing them into the 'high' or 'low' risk genotype $[18,19]$. To accomplish this, GMDR calculates 'score' using the target phenotype (i.e., the urinary IsoP level in this case) and covariates. If the sum of the score for a particular genotype set exceeds a threshold, the genotype set is categorized 'high risk' and vice versa [16]. GMDR selects the best reproducible combination of SNPs to categorize high and low risk genotypes using the cross-validation strategy, and statistical significance of the model is examined by a permutation test. In a permutation test, a particular data set used in a study is used. The score data are shuffled randomly to construct a new 'virtual' data set that is tested by GMDR. This procedure is repeated 1000 times to obtain an adequate empirical significant level in the particular data set. Correction for multiple testing is therefore not necessary in this case.

In the present study, because the averaged IsoP level differed significantly between the two populations, the IsoP level of individual subject was standardized in each county and GMDR was performed on the combined population. Covariates used in the calculation of the score were sex, age, low-density lipoprotein cholesterol (LDL-C), log HOMA-R, eGFR, the presence of
DM, and the smoking and the drinking histories, all of which showed independent effects on the IsoP level at $p<0.1$ in the initial linear regression analysis. Statistical significance of the models was examined in a 1000-times permutation test.

\section{Results}

Table 1 summarizes the demographic data of the two populations studied. The subjects from M county were significantly younger than those from $\mathrm{K}$ county. Of interest, The IsoP level was greater in M county than in K county. HOMA-R, eGFR, high-density lipoprotein cholesterol (HDL-C), TG and the incidence of regular drinkers were greater, and incidence of hypertensives with medication were less in M county.

Genotype and allele frequencies of the SNPs examined were shown in Table 2. All the genotype frequencies were in Hardy-Weinberg equilibrium. The genotype and allele frequencies were not significantly different between the two regions studied. Allele frequencies of the four SNPs in the p22phox, eNOS, LpPLA2 and PON1 genes in these populations were similar to those in the public database (www.ncbi.nim.nih.gov/ projects/SNP) and/or in the previous reports [20-22]. In contrast, the both populations had substantially 
Table 2

Genotype and allele frequencies of 6 SNPs studied

\begin{tabular}{|c|c|c|c|c|}
\hline & & K county & M county & $\mathrm{p}^{*}$ \\
\hline \multirow{5}{*}{$\begin{array}{l}\text { LpPLA2, G994T } \\
\text { (rs16874954) }\end{array}$} & GG & 467 & 253 & \multirow[t]{3}{*}{0.6} \\
\hline & GT & 185 & 119 & \\
\hline & $\mathrm{TT}$ & 26 & 17 & \\
\hline & G & 0.825 & 0.803 & \multirow[t]{2}{*}{0.4} \\
\hline & $\mathrm{T}$ & 0.175 & 0.197 & \\
\hline \multirow{5}{*}{$\begin{array}{l}\text { eNOS, T-786C } \\
\text { (rs2070744) }\end{array}$} & $\mathrm{TT}$ & 517 & 313 & \multirow[t]{3}{*}{0.9} \\
\hline & $\mathrm{CT}$ & 145 & 87 & \\
\hline & $\mathrm{CC}$ & 11 & 4 & \\
\hline & $\mathrm{T}$ & 0.876 & 0.882 & \multirow[t]{2}{*}{0.9} \\
\hline & $\mathrm{C}$ & 0.124 & 0.118 & \\
\hline \multirow{5}{*}{$\begin{array}{l}\text { MTHFR, C677T } \\
\text { (rs1801133) }\end{array}$} & $\mathrm{CC}$ & 184 & 112 & \multirow[t]{3}{*}{0.7} \\
\hline & $\mathrm{CT}$ & 345 & 221 & \\
\hline & $\mathrm{TT}$ & 143 & 75 & \\
\hline & $\mathrm{C}$ & 0.531 & 0.545 & 0.9 \\
\hline & $\mathrm{T}$ & 0.469 & 0.455 & \\
\hline \multirow{5}{*}{$\begin{array}{l}\text { p22phox, C242T } \\
\text { (rs4673) }\end{array}$} & $\mathrm{CC}$ & 543 & 317 & \multirow[t]{3}{*}{0.3} \\
\hline & $\mathrm{CT}$ & 127 & 69 & \\
\hline & $\mathrm{TT}$ & 6 & 0 & \\
\hline & $\mathrm{C}$ & 0.897 & 0.911 & \multirow[t]{2}{*}{0.6} \\
\hline & $\mathrm{T}$ & 0.103 & 0.089 & \\
\hline \multirow{5}{*}{$\begin{array}{l}\text { PON1, Q192R } \\
\text { (rs662) }\end{array}$} & $\mathrm{RR}$ & 292 & 192 & \multirow[t]{3}{*}{0.3} \\
\hline & QR & 312 & 169 & \\
\hline & QQ & 63 & 47 & \\
\hline & $\mathrm{R}$ & 0.672 & 0.678 & 1.0 \\
\hline & Q & 0.328 & 0.322 & \\
\hline \multirow{5}{*}{$\begin{array}{l}\text { PON1, T-107C } \\
\text { (rs705379) }\end{array}$} & $\mathrm{TT}$ & 212 & 130 & \multirow[t]{3}{*}{0.8} \\
\hline & $\mathrm{CT}$ & 318 & 183 & \\
\hline & $\mathrm{CC}$ & 140 & 96 & \\
\hline & $\mathrm{T}$ & 0.554 & 0.542 & \multirow[t]{2}{*}{0.9} \\
\hline & $\mathrm{C}$ & 0.446 & 0.458 & \\
\hline
\end{tabular}

* Allele and genotype frequencies of each SNP were compared between the $\mathrm{K}$ and $\mathrm{M}$ county by the contingency table analysis.

greater frequency of the minor allele for MTHFR C677T, when compared with those in a previous report on Japanese [23]. This discrepancy may be explained by the fluctuation due to a small size of the population examined in the previous study or by a regional drift in the allele frequency; the population employed in this study is from a relatively isolated mountainous region with little population movement. Anyway, the genotypes examined in the two independent populations gave a similar allele frequencies for all the six SNPs, which suggested that the allele frequencies obtained in the present study were reliable.

Age was significantly correlated with IsoP in a univariate analysis $(r=0.082, p=0.03$ and $r=0.13$, $p=0.009$ in $\mathrm{K}$ and $\mathrm{M}$ counties, respectively). HDL-C, LDL-C and eGFR showed modest but significant correlations with IsoP level in one of the two populations (data not shown). None of the SNPs had significant effects on the IsoP level either in the two populations by ANOVA (data not shown).

As the significant effects of the SNPs were not detected in the initial analysis, the GMDR analysis was performed on the combined $\mathrm{K}$ and $\mathrm{M}$ population to examine the combined effects of the 6 SNPs. As shown in Table 3, permutation tests indicated that combination of the two (eNOS T-786C and MTHFR C677T) and of the three SNPs (eNOS T-786C, MTHFR C677T and LpPLA2 G994T) gave significant and marginally significant effects on the IsoP level ( $p=0.033$ and 0.076 , respectively). Other combinations did not show significant effects on the IsoP level.

The panel A and B of Fig. 1 show combinations of the genotype categorized as 'high' and 'low' risk for the two SNPs model (eNOS - MTHFR) and for the three SNPs model (eNOS - MTHFR - Lp-PLA2), respectively, and their effects on the IsoP level in the two populations (the right panels of Fig. 1).

Multiple linear regression models were constructed including the results of GMDR analysis (Table 4). Both combinations of the 2 and 3 SNPs remained to be independent factors influencing the IsoP level after adjustment of potential confounding factors.

\section{Discussion}

In this study, effects of 6 functional SNPs on the isoP level were examined in two general populations in a rural area of Japan. The urinary IsoP level is thought to be a good marker for the oxidative stress in population studies because IsoP is a stable substance in the urine even under the room temperature [11]. Further, several reports indicated that the urinary IsoP level was a risk factor for cardiovascular diseases, such as cerebrovascular disease and myocardial infarction $[13,14]$.

Six SNPs examined here were indicated to influence either the amount of enzymes (PON1 T-107C, eNOS T-786C and MTHFR C677T) or the enzyme activity (PON1 Q192R, LpPLA2 G994T and p22phox $\mathrm{C} 242 \mathrm{~T}$ ) [5-10]. As these enzymes may regulate the oxidative stress either directly (p22phox [24]) or indirectly through generating and degrading anti-oxidative and oxidative substances such as oxidized lipids (PON1 and LpPLA2 [25,26]), nitric oxide (eNOS [6]) and homocysteine (MTHFR [27]), the SNPs examined here were good candidates for potential regulators of the oxidative stress in vivo.

In spite of such expectation, the results of the present study indicated that these SNPs had no major independent effects on the oxidative stress in general populations. This may be due to small allelic effects of the SNPs on the isoP level; as the oxidative stress is regulated by complex mechanisms in which many molecules 
Table 3

Summary of GMDR analysis

\begin{tabular}{|c|c|c|c|c|}
\hline No. of SNPs combined & The best model & Test accuracy & $\mathrm{CVC}$ & $\mathrm{p}$ \\
\hline 2 & eNOS $\cdot$ MTHFR & 0.548 & 8 & 0.033 \\
\hline 3 & LpPLA2 $\cdot$ eNOS $\cdot$ MTHFR & 0.538 & 8 & 0.076 \\
\hline 4 & LpPLA2 · eNOS · MTHFR · PON1 Q192R & 0.506 & 5 & 0.42 \\
\hline 5 & LpPLA2 $\cdot$ eNOS $\cdot$ MTHFR $\cdot$ PON1 Q192R $\cdot$ PON1 T-107C & 0.492 & 6 & 0.58 \\
\hline 6 & All & 0.476 & 4 & 0.76 \\
\hline
\end{tabular}

CVC; cross-validation consistency. $\mathrm{P}$ values were obtained through a permutation test (1000 times permutation).

A
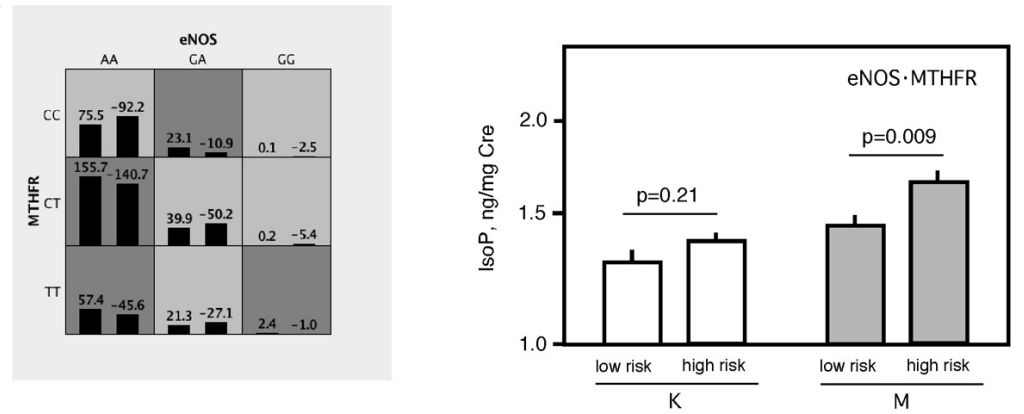

B

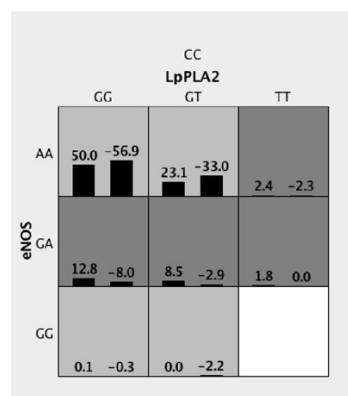

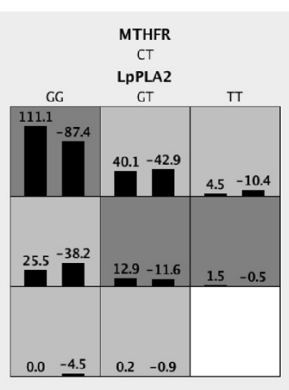

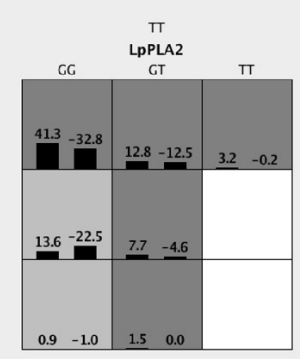

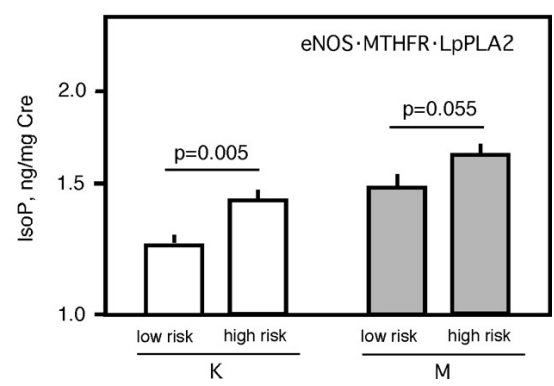

Fig. 1. The summary of GMDR analysis. Effects of the combination of the two SNPs (MTHFR C677T and eNOS T-786C) and of the three SNPs (MTHFR C677T, eNOS T-786C and LpPLA2 G994T) were summarized in the panel (A) and (B), respectively. Columns and numbers above the columns indicated the sum of 'positive' and 'negative' scores in each cell (= each combination of genotypes). Positive and negative scores represent above and below the averaged score $(=0)$, respectively. Cells (= genotype combinations) are categorized into 'high' and 'low' risk when the sum of the score is above and below zero, respectively. For example, in the panel (A), the combination of AA (eNOS) and CC (MTHFR) is categorized as 'low risk' because the sum of the score is below zero $(75.5-92.2<0)$. Cells in dark and light gray indicate a 'high' and 'low' risk combination of the genotypes, respectively. Cells in white are empty. IsoP levels in the 'high' and 'low' risk genotypes were compared in the two populations in the right panels.

are involved [3], contribution of each enzyme might be small or, in another word, functional deficiency in one enzyme might be compensated by another. Among the six genes examined, the T allele of the p22phox C242T showed a substantial recessive effect on the IsoP level although it did not reach a significant level (CC; $1.31 \pm$ 0.03 , CT; $1.48 \pm 0.09$, TT; $0.99 \pm 0.29 \mathrm{ng} / \mathrm{mg}$ Cre, $p=0.06$, in the subjects of $\mathrm{K}$ county). This negative observation might be due to a low frequency of the TT homozygotes even though we employed 1000 subjects. Using more subjects may be able to establish a significant role of this SNP on the IsoP level.
In contrast to the analysis of effects of individual SNPs, GMDR analysis identified the combinations of SNPs that significantly influenced the IsoP level. GMDR was introduced by Lou et al. as an extended version of MDR [16], which could reduce the complexity substantially $[16,18,19]$. Further, GMDR has additional advantages over MDR; GMDR can deal with quantitative phenotypes and with unbalanced case-control samples [16]. These aspects of GMDR made it possible to analyze a quantitative phenotype under a crosssectional study design. With GMDR, all of 57 sets of the 6 SNPs (15 pairs, 20 trios, 15 quartets, 6 quintets 
Table 4

Multiple linear regression models for the IsoP level including the SNP combination (A) eNOS · MTHFR

\begin{tabular}{lccccc}
\hline & $\beta$ & $\mathrm{SE}$ & Standardized $\beta$ & $\mathrm{t}$ & $\mathrm{p}$ \\
\hline region, M county & 0.031 & 0.0086 & 0.12 & 3.63 & 0.0003 \\
sex, female & 0.034 & 0.010 & 0.13 & 3.22 & 0.001 \\
age & $3.3 \times 10^{-3}$ & $9.8 \times 10^{-4}$ & 0.12 & 3.35 & 0.0008 \\
regular drinker & 0.023 & 0.012 & 0.07 & 1.96 & 0.05 \\
log HOMA-R & 0.053 & 0.024 & 0.07 & 2.23 & 0.03 \\
LDL-C & $-8.1 \times 10^{-4}$ & $3.1 \times 10^{-4}$ & -0.08 & -2.64 & 0.01 \\
eGFR & $1.1 \times 10^{-3}$ & $5.4 \times 10^{-4}$ & 0.07 & 2.09 & 0.04 \\
SNPs, high risk & 0.020 & 0.008 & 0.08 & 2.47 & 0.01 \\
\hline
\end{tabular}

$\mathrm{PR}^{2}$ adjusted for the degrees of freedom $=0.050$.

(B) eNOS $\cdot$ MTHFR $\cdot$ LpPLA2

\begin{tabular}{lccccc}
\hline & $\beta$ & $\mathrm{SE}$ & Standardized $\beta$ & $\mathrm{t}$ & $\mathrm{p}$ \\
\hline region, M county & 0.032 & 0.0086 & 0.12 & 3.77 & 0.0002 \\
sex, female & 0.032 & 0.010 & 0.12 & 3.08 & 0.002 \\
age & $3.3 \times 10^{-3}$ & $9.8 \times 10^{-4}$ & 0.12 & 3.35 & 0.0008 \\
regular drinker & 0.021 & 0.012 & 0.07 & 1.82 & 0.07 \\
log HOMA-R & 0.052 & 0.024 & 0.07 & 2.18 & 0.03 \\
LDL-C & $-8.2 \times 10^{-4}$ & $3.1 \times 10^{-4}$ & -0.09 & -2.64 & 0.008 \\
eGFR & $1.1 \times 10^{-3}$ & $5.4 \times 10^{-4}$ & 0.07 & 2.13 & 0.04 \\
SNPs, high risk & 0.027 & 0.008 & 0.11 & 3.49 & 0.0005 \\
\hline
\end{tabular}

$\mathrm{R}^{2}$ adjusted for the degrees of freedom $=0.056$.

and 1 sextet) were analyzed in this study, which is not practical when a parametric analysis is used.

As pointed out by Ritchie et al., however, it is often difficult to deduce underlying physiological mechanisms how the genotypes interact to (and not to) impose effects on the target phenotypes [18]. In the present case, it is difficult as well to interpret how the combination of the SNPs in the eNOS, MTHFR and LPPLA2 gene affect the isoP level. The homocysteine level was reported to be associated with the level of asymmetric dimethlyarginine (ADMA) [28]. As ADMA is an endogenous inhibitor of NOS [29], this might explain the combined effect of these SNPs. Meanwhile, LpPLA2 might contribute to the IsoP level through releasing this substance from the oxidized phospholipids [30].

Recent advance in large-scale genome-wide association analyses identified many SNPs relating to multifactorial diseases or affecting quantitative phenotypes [31, 32]. In spite of that, the effects of SNPs are often too small to explain total genetic variance even if all the effects are additively combined [33,34]. In this context, it is necessary to investigate gene-gene and geneenvironmental interactions to elucidate whether they can complement the small effects of SNPs [35]. The present study suggested that GMDR is one of promising analytical methods to be applied in such studies.

The linear regression analysis confirmed that the combinations of SNPs were independent factors influencing the IsoP level (Table 4). From the point of epidemiological view, however, the effects were quite small, and further, the regression model itself explained only a small part of the variation of the IsoP level. This may suggest that other unknown factors have major effects on the IsoP level. We have to point out, however, that IsoP was measured in spot urine samples in this study, which might be more susceptible to environmental noises. Better strategies for the urine collection such as 24-hour urine collection and/or better markers for oxidative stress are necessary to be employed to solve this problem. In addition, the present results need to be confirmed in another large population.

It is of interest that there was a clear difference in the IsoP level between the two counties. The result of the multiple linear regression analysis indicated that this regional difference was significant even after several confounding factors were adjusted (see Table 4). This implied that other unknown factors such as food, social capitals and physical activities, affected the IsoP level in the two counties. As the two counties are closely located with each other in a mountainous area of Japan, it is interesting what difference in the life style might be responsible for this observation. Further investigations on this issue are warranted.

\section{Acknowledgement}

This study was partially supported by a grant-in-aid for scientific research of the Ministry of Education, 
Sports, Science and Techonology, Japan, and by a grant of the Research Project Promotion Institute, Shimane University.

\section{References}

[1] D.D. Heistad, Oxidative stress and vascular diseases, Arterioscler Thromb Vasc Biol 26 (2006), 689-695.

[2] M.C. Houston, The role of cellular micronutrient analysis, nutraceuticals, vitamins, antioxidants and minerals in the prevention and treatment of hypertension and cardiovascular disease, Ther Adv Cardiovasc Dis 4 (2010), 165-183.

[3] A.L. Levonen, E. Vähäkangas, J.K. Koponen and S. YläHerttuala, Antioxidant gene therapy for cardiovascular disease: current status and future perspectives, Circulation 117 (2008), 2142-2150.

[4] J.W. Stephens, S.C. Bain and S.E. Humphries, Geneenvironment interaction and oxidative stress in cardiovascular disease, Atherosclerosis 200 (2008), 229-238.

[5] V.H. Brophy, R.L. Jampsa, J.B. Clendenning, L.A. McKinstry, G.P. Jarvik and C.E. Furlong, Effects of 5' regulatory-region polymorphisms on paraoxonase-gene (PON1) expression, Am J Hum Genet 68 (2001), 1428-1436.

[6] M. Nakayama, H. Yasue, M. Yoshimura, Y. Shimasaki, K. Kugiyama, H. Ogawa, T. Motoyama, Y. Saito, Y. Ogawa, Y. Miyamoto and K. Nakao, T-786->C mutation in the 5'flanking region of the endothelial nitric oxide synthase gene is associated with coronary spasm, Circulation 99 (1999), 2864 2870 .

[7] D.M. Stafforini, K. Satoh, D.L. Atkinson, L.W. Tjoelker, C. Eberhardt, H. Yoshida, T. Imaizumi, S. Takamatsu, G.A. Zimmerman, T.M. McIntyre, P.W. Gray and S.M. Prescott, Platelet-activating factor acetylhydrolase deficiency, A missense mutation near the active site of an anti-inflammatory phospholipase, J Clin Invest 97 (1996), 2784-2791.

[8] T.J. Guzik, N.E. West, E. Black, D.McDonald, C. Ratnatunga, R. Pillai and K.M. Channon, Functional effect of the C242T polymorphism in the NAD $(\mathrm{P}) \mathrm{H}$ oxidase $\mathrm{p} 22$ phox gene on vascular superoxide production in atherosclerosis, Circulation 102 (2000), 1744-1747.

[9] C. Cahilly, C.M. Ballantyne, D.S. Lim, A. Gotto and A.J. Marian, A variant of p22(phox), involved in generation of reactive oxygen species in the vessel wall, is associated with progression of coronary atherosclerosis, Circ Res 86 (2000), 391-395.

[10] L. Brattström, D.E. Wilcken, J.Ohrvik and L. Brudin, Common methylenetetrahydrofolate reductase gene mutation leads to hyperhomocysteinemia but not to vascular disease: the result of a meta-analysis, Circulation 98 (1998), 2520-2526.

[11] J.D. Morrow, Y. Chen, C.J. Brame, J. Yang, S.C. Sanchez, J. Xu, W.E. Zackert, J.A. Awad and L.J. Roberts, The isoprostanes: unique prostaglandin-like products of free-radicalinitiated lipid peroxidation, Drug Metab Rev 31 (1999), 117139.

[12] J. Nourooz-Zadeh, Key issues in F2-isoprostane analysis, Biochem Soc Trans 36 (2008), 1060-1065.

[13] H. Shibata, T. Nabika, H. Moriyama, J. Masuda and S. Kobayashi, Correlation of NO metabolites and 8-isoprostaglandin F2a with periventricular hyperintensity severity, Arteiroscler Thromb Vasc Biol 24 (2004), 1659-1663.

[14] Y. Jang, O.Y. Kim, S.J. Koh, J.S. Chae, Y.G. Ko, J.Y. Kim, H. Cho, T.S. Jeong, W.S. Lee, J.M. Ordovas and J.H. Lee,
The Val279Phe variant of the lipoprotein-associated phospholipase A2 gene is associated with catalytic activities and cardiovascular disease in Korean men, J Clin Endocrinol Metab 9 (2006), 3521-3527.

[15] A. Upadhyay, M.G. Larson, C.Y. Guo, R.S. Vasan, I. Lipinska, C.J. O’Donnell, S. Kathiresan, J.B. Meigs, J.F. Keaney J. Jr, Rong, E.J. Benjamin and C.S. Fox, Inflammation, kidney function and albuminuria in the Framingham Offspring cohort, Nephrol Dial Transplant 25 (2010), in press.

16] X.Y. Lou, G.B. Chen, L. Yan, J.Z. Ma, J. Zhu, R.C. Elston and M.D. Li, A generalized combinatorial approach for detecting gene-by-gene and gene-by-environment interactions with application to nicontine dependence, Am J Hum Genet 80 (2007), 1125-1137.

[17] S. Matsuo, E. Imai, M. Horio, Y. Yasuda, K. Tomita, K. Nitta, K. Yamagata, Y. Tomino, H. Yokoyama and A. Hishida, Collaborators developing the Japanese equation for estimated GFR, Revised equations for estimated GFR from serum creatinine in Japan, Am J Kidney Dis 53 (2009), 982-992.

[18] M.D. Ritchie, L.W. Hahn, N. Roodi, L.R. Bailey, W.D. Dupont, F.F. Parl and J.H. Moore, Multifactor-dimensionality reduction reveals high-order interactions among estrogenmetabolism genes in sporadic breast cancer, Am J Hum Genet 69 (2001), 138-147.

[19] L.W. Hahn, M.D. Ritchie and J.H. Moore, Multifactor dimensionality reduction software for detecting gene-gene and geneenvironmant interactions, Bioinformatics 19 (2003), 376-382.

[20] S. Nasreen, T. Nabika, H. Shibata, H. Moriyama, K. Yamashita, J. Masuda and S. Kobayashi, T-786C polymorphism in endothelial NO synthase gene affects cerebral circulation in smokers: possible gene-environmental interaction, Arterioscler Thromb Vasc Biol 22 (2002), 605-610.

[21] A. Imamura, R. Takahashi, R.Murakami, H. Kataoka, X.W. Cheng, Y. Numaguchi, T. Murohara and K. Okumura, The effects of endothelial nitric oxide synthase gene polymorphisms on endothelial function and metabolic risk factors in healthy subjects: the significance of plasma adiponectin levels, Eur J Endocrinol 158 (2008), 189-195.

[22] T. Suehiro, T. Nakamura, M. Inoue, T. Shiinoki, Y. Ikeda, Y. Kumon, M. Shindo, H. Tanaka and K. Hashimoto, A polymorphism upstream from the human paraoxonase (PON1) gene and its association with PON1 expression, Atherosclerosis 150 (2000), 295-298.

[23] M. Iemitsu, H. Murakami, K. Sanada, K. Yamamoto, H. Kawano, Gando and M. Miyachi, Lack of carotid stiffening associated with MTHFR 677TT genotype in cardiorespiratory fit adults, Physiol Genomics 42 (2010), 259-265.

[24] B. Lassegue and K.K. Griendling, NADPH oxidases: functions and pathologies in the vasculature, Arterioscler Thromb Vasc Biol 30 (2010), 653-661.

[25] A.D. Watson, J.A. Berliner, S.Y. Hama, B.N. La Du, K.F. Faull, A.M. Fogelman and M. Navab, Protective effect of high density lipoprotein associated paraoxonase: inhibitionof the biological activity of minimally oxidized low density lipoprotein, J Clin Invest 96 (1995), 2882-2891.

[26] T. Wang, K. Karino, M. Yamasaki, Y. Zhang, J. Masuda, S. Yamaguchi, K. Shiwaku and T. Nabika, Effects of G994T in the Lp-PLA2 gene on the plasma oxidized LDL leel and carotid intima-media thickness in Japanese: The Shimane Study, Am J Hypertens 22 (2009), 742-747.

[27] D. Gemmati, M. Previati, M.L. Serino, S. Moratelli, S. Guerra, S. Capitani, E. Forini, G. Ballerini and G.L. Scapoli, Low folate levels and thermolabile methylenetetrahydrofolate reductase as primary determinant of mild hyperhomocystinemia 
in normal and thromboembolic subjects, Arterioscler Thromb Vasc Biol 19 (1999), 1761-1767.

[28] R.H. Böger, S.M. Bode-Böger, K. Sydow, D.D. Heistad and S.R. Lentz, Plasma concentration of asymmetric dimethylarginine, an endogenous inhibitor of nitric oxide synthase, is elevated in monkeys with hyperhomocyst(e)inemia or hypercholesterolemia, Arterioscler Thromb Vasc Biol 20 (2000), 1557-1564.

[29] C. Zoccali, ADMA: a cardiovascular and renal risk factor on the move, J Hypertens 24 (2006), 611-619.

[30] D.M. Stafforini, J.R. Sheller, T.S. Blackwell, A. Sapirstein, F.E. Yull, T.M. McIntyre, J.V. Bonventre, S.M. Prescott and L.J. Roberts II, Release of gree F2-isoprostanes from esterified phospholipids is catalyzed by intracellular and plasma plateletactivating factor acetylhydrolases, J Biol Chem 281 (2006), 4616-4623.

[31] Wellcome Trust Case Control Consortium, Genome-wide association study of 14,000 cases of seven common diseases and
3,000 shared controls, Nature 447 (2007), 661-678.

[32] Y.S. Cho, M.J. Go, Y.J. Kim, J.Y. Heo, J.H. Oh, H.J. Ban, D. Yoon, M.H. Lee, D.J. Kim, M. Park, S.H. Cha, J.W. Kim, B.G. Han, H. Min, Y. Ahn, M.S. Park, H.R. Han, H.Y. Jang, E.Y. Cho, J.E. Lee, N.H. Cho, C. Shin, T. Park, J. W.Park, J. K.Lee, L. Cardon, G. Clarke, M.I. McCarthy, J.Y. Lee, J.K. Lee, B. Oh and H.L. Kim, A large-scale genome-wide association study of Asian populations uncovers genetic factors influencing eight quantitative traits, Nat Genet 41 (2009), 527-534.

[33] D.B. Goldstein, Common genetic variation and human traits, N Engl J Med 360 (2009), 1696-1698.

[34] G. Gibson, Hints of hidden heritability in GWAS, Nat Genet 42 (2010), 558-560.

[35] R.J. Neuman, J. Wasson, G. Atzmon, J. Wainstein, Y. Yerushalmi, J. Cohen, N. Barzilai, I. Blech, B. Glaser and M.A. Permutt, Gene-gene interactions lead to higher risk for development of type 2 diabetes in an Ashkenazi Jewish population, PLoS One 5 (2010), e9903. 


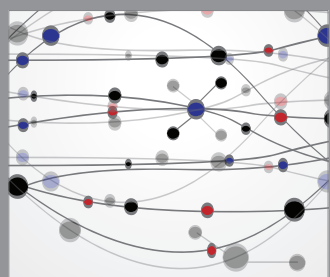

The Scientific World Journal
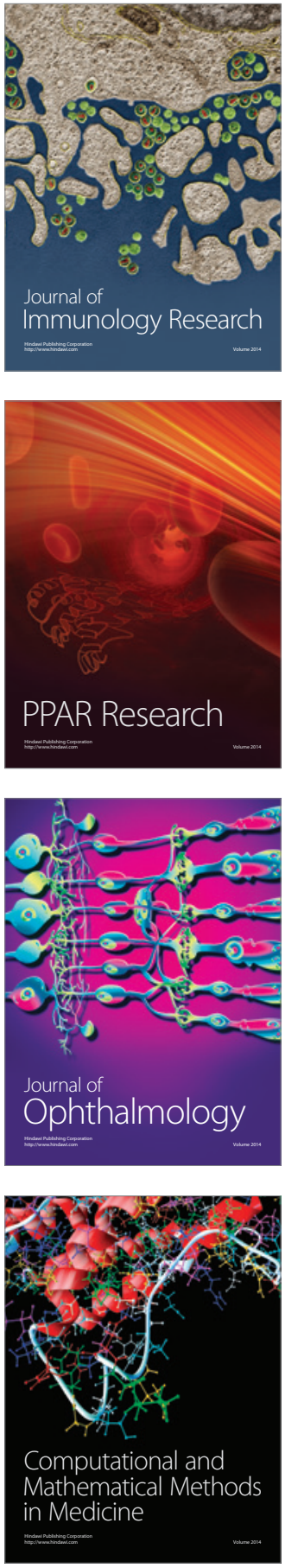

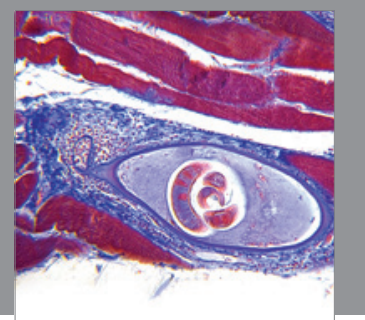

Gastroenterology

Research and Practice
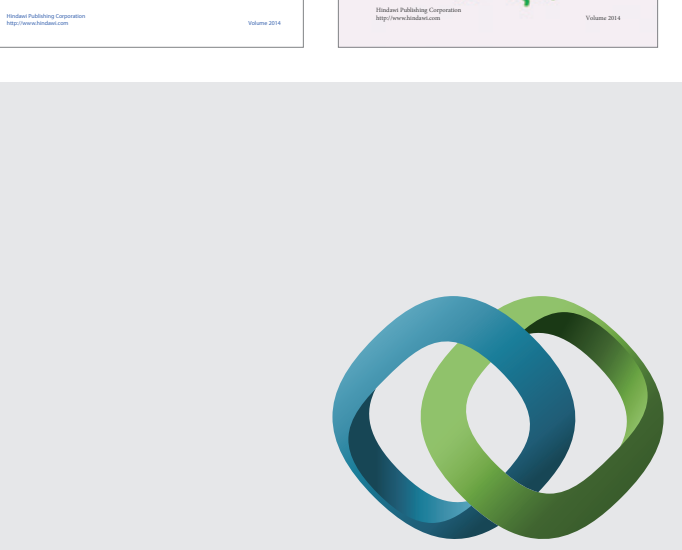

\section{Hindawi}

Submit your manuscripts at

http://www.hindawi.com
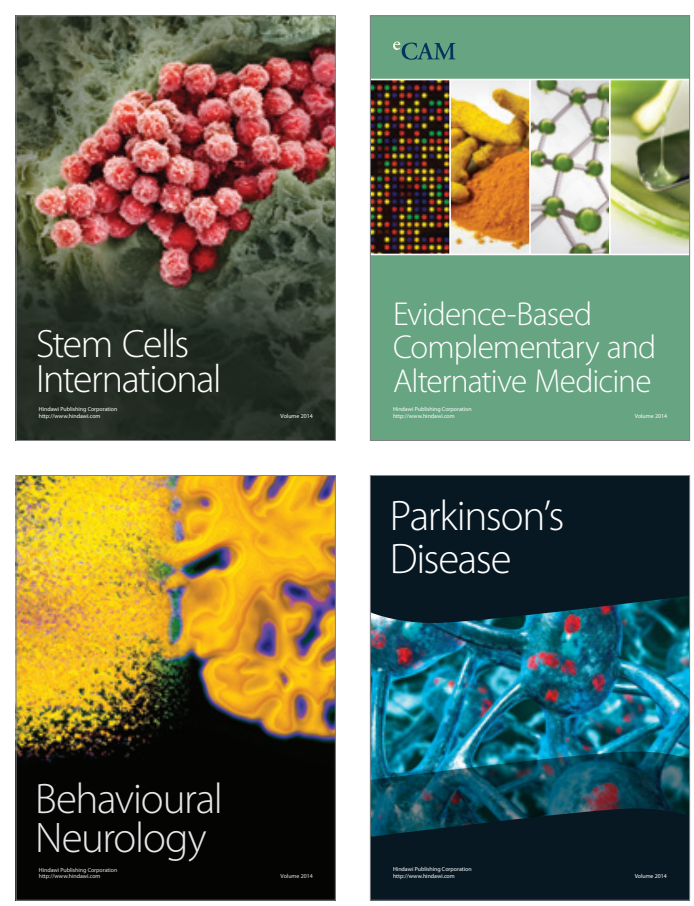

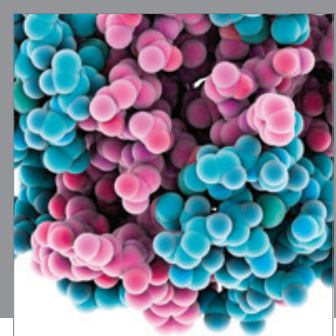

Journal of
Diabetes Research

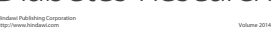

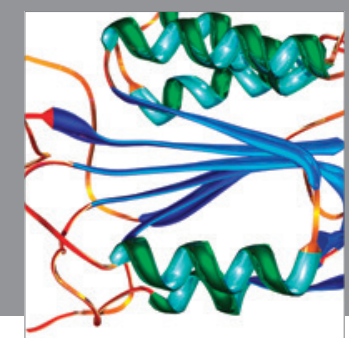

Disease Markers
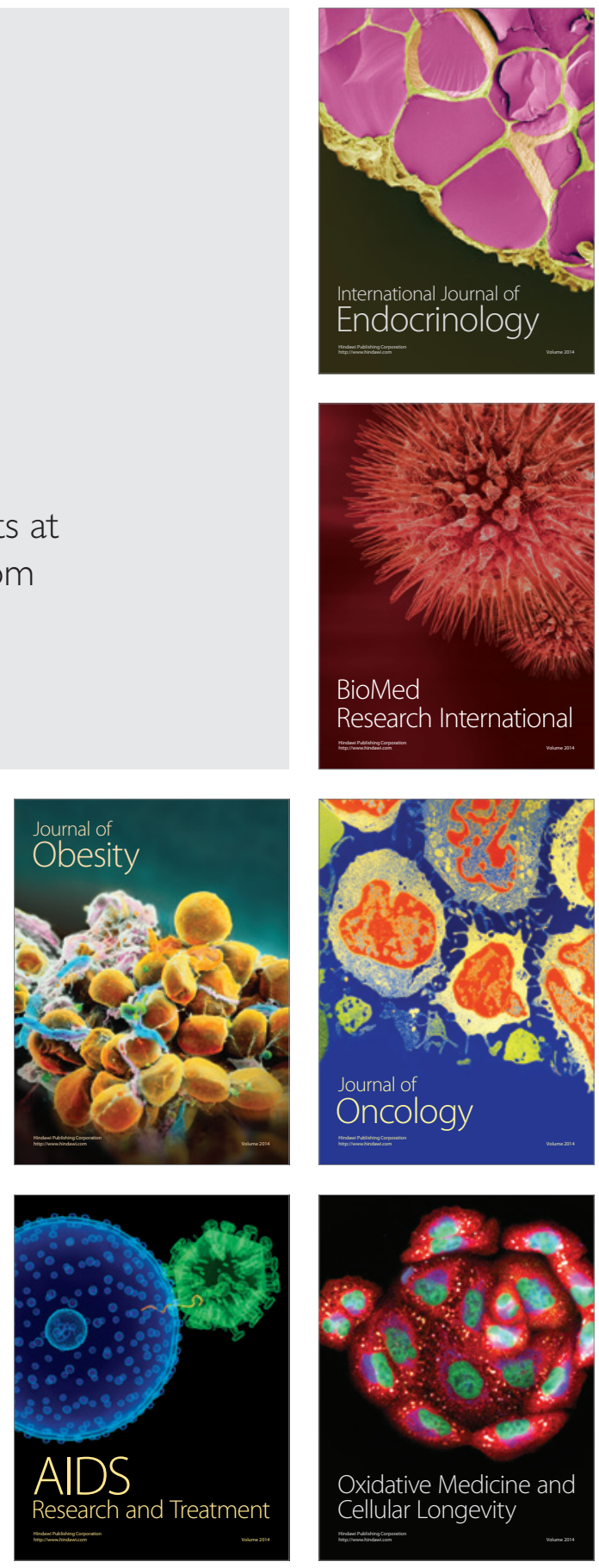